\title{
Towards Distributed Diagnosis of The Tennessee Eastman Process Benchmark
}

\author{
Jun Chen and John Howell* \\ Department of Mechanical Engineering \\ University of Glasgow \\ Glasgow G12 8QQ \\ Scotland, UK
}

Suggested running title: Towards distributed diagnosis

\begin{abstract}
A distributed hybrid strategy is outlined for the isolation of faults and disturbances in the Tennessee Eastman Process, which would build on existing structures for distributed control systems, so should be easy to implement, be cheap and be widely applicable. The main emphasis in the paper is on one component of the strategy, a steady state based approach. Results obtained by applying this approach are presented and knowledge limitations are discussed. In particular a way in which a knowledge-base might evolve to improve isolation capabilities is suggested and the role of the operator is briefly discussed.
\end{abstract}

Keywords: process control, fault diagnosis, distributed control systems

\section{Introduction}

Nowadays, feedback control loop components can be smart and have selfcomputational capabilities linked by a high-speed, bi-directional data communication bus that allows the central supervisory system to interrogate devices for status and other information. If one looks at the measurements collected from a continuously operated process plant, a large proportion relate to control loops, the rest are largely collected to ensure that operation is within allowable constraints. Since these control loops are distributed throughout the plant, it seems sensible to examine the possibility of distributing associated detection \& diagnosis tasks with them. These tasks would aim to isolate faults and disturbances in both the control loop and in that part of the process that impinges on control loop performance. Economies of scale would then be achieved by making use of common software to configure the tasks at the same time as individual loops were tuned. Clearly these economies of scale would be diminished if the algorithms were too plant specific and required knowledge not readily available from the plant. Fig. 1 shows the kind of implementation envisaged: the SEVACS (self-validating control systems) modules would perform detection \& diagnostic tasks. The term SEVACS was chosen to highlight a certain similarity with the SEVA concept (Clarke, 1995), which seeks to distribute local detection \& diagnosis tasks to instruments and actuators. The main conceptual difference is that

\footnotetext{
* To whom all correspondence should be addressed. Email: j.howell@mech.gla.ac.uk. Fax: +44 (0) 141 3304343
} 
part of the process is covered also. The boundaries specified for individual SEVACS module responsibilities should overlap one another and their union should encompass the entire plant. This overlapping means that complete distribution will not be achieved because individual SEVACS conclusions need to be correlated. Comparison would be performed by the FDD Supervisor, which would also perform centralised detection \& diagnostic tasks and provide an interface with the user. In a previous paper Chen \& Howell (2001) have described one possible approach to designing SEVACS modules. By focusing on an application, the Tennessee Eastman (T-E) Process Benchmark, this paper seeks to demonstrate this design methodology and to identify the additional functions that are needed to produce a complete capability. The T-E benchmark is a particularly appropriate choice, because the proposed approach is intended to be used to isolate incipient faults and disturbances in plants with control loops that inherently eliminate steady state error, and control loops in the Benchmark possess this capability.

The motivation for this research stems from the fact that although a considerable amount has been published about detection algorithms that could be distributed, little has been published about algorithms, to isolate ${ }^{1}$ a fault or major disturbance, which can be distributed. Although a number of detection algorithms might be sufficiently local to locate a specific fault, in general less is available to isolate (Chow \& Willsky, 1984; Basseville \& Nikiforov, 1993; Gertler, 1993, 1998; Himmelblau, 1978; Isermann, 1984, 1997). Based on qualitative reasoning, the proposed approach has been developed, in part, by referring to SDG representations of control systems and their interactions. Although the application of SDG-based reasoning to fault diagnosis is not new, previous work has focused on the process plant, with its associated control and sensory systems, as a single entity (e.g. Iri et al., 1979; Shiozaki et al., 1984; Tsuge et al., 1984a, 1984b; Kramer \& Palowitch, 1987; Kutsuwa, Kojima \& Matsuyama, 1988; Mo et al., 1997). Although not directly relevant because the approach cannot be distributed, it is interesting that Vedam and Venkatasubramanian (1999) have developed a hybrid approach based on PCA and SDG. Wang et al. (1995) have applied fuzzy qualitative reasoning method to assess process plants whilst Lunze and Schiller (1999) have explored fault diagnosis based on qualitative and probabilistic logic models. There also have been a number of papers published recently on control loop performance monitoring (Harris, 1989; Thornhill et al., 1996, 1999; Tyler \& Morari, 1996). Nevertheless its aim is to assess the behaviour of the control loop itself such as whether the control loop works in a good or optimal condition, and whether tuning parameters (e.g., P, I or D) are satisfied. The method itself is immune to load disturbances and it lacks the ability to isolate faults. Finally the reader is referred to Chantler, Coghill, Shen, \& Leitch (1998), which is considered noteworthy because it outlines various implementations that have been examined in realistic situations.

The paper has five main sections. The steady state approach is outlined in Section 2 and this is followed, in Section 3, by a brief description of other components of the strategy. Section 4 then describes how these might be applied to the Benchmark. Section 5 discusses some results and conclusions are drawn in Section 6.

\footnotetext{
${ }^{1}$ Fault isolation: determination of the kind, location and time of detection of a fault (Isermann, R. \& Balle, P., 1997).
} 


\section{The Distributed Steady State Approach}

The steady state approach has two components, a distributed component and a central component. In the distributed component candidate sets of faults and disturbances are hypothesised by reasoning qualitatively about how steady state deviations, observed in the control system, might have been caused. This reasoning process is based on qualitative equations derived for that particular form of control system. Two standard forms are considered briefly in Section 2.1: a single loop control system and a cascade control system. The reader is referred to Chen \& Howell (2001) and Chen (2000) for further details. As will be seen when describing the T-E application, non-standard forms can be analysed in a similar way. In the central component the candidate sets generated by the various SEVACS are then fused by applying various isolation procedures, all of which take into account known interactions between control systems and sign information output from the SEVACS. These procedures are outlined in Section 2.2.

The steady state approach would be implemented in two stages, as part of the (offline) design stage and then, online, during commissioning. At the offline stage the plant would be decomposed into manageable compartments and each control system would be considered in turn. The following characteristics would then be identified/hypothesised for each control system:

- its structure, i.e. whether it has a standard form like a single loop or double, cascade loops or whether a new special form has to be recognised;

- the process Type Number (Dorf \& Bishop, 1995) (if known);

- open loop stability (if known);

- steady state gains between interacting loops (if known).

Based on these characteristics, an appropriate configuration would then be downloaded to the SEVACS. During commissioning, online procedures would then be executed to obtain those items that were still unknown. These previously unpublished, on-line procedures are outlined in Section 2.3. In addition the SEVACS would be configured to detect changes in steady state (see Chen \& Howell, 2001) and the FDD supervisor would be specified.

\subsection{Analysis of The Standard Forms}

Consider the standard single loop control system shown in Fig. 2 where various variables represent steady state deviations: $\theta_{r}$ is the deviation in the set-point/reference variable, $\theta$ in the controlled variable, e in the control error, $d_{m}$ in the sensor bias, $d_{v}$ in the valve bias, $d_{p}$ in the process disturbance, $\mathrm{x}$ in the controller output. Parameter $K_{c}$ is the steady state proportional gain of the controller and parameters $K_{v}, K_{p}$ and $K_{d}$ are respectively the valve, process and process disturbance steady state gains. Transfer functions $G_{c}(s), G_{v}(s), G_{p}(s)$ and $G_{d}(s)$ are for the controller, valve, process and disturbance respectively.

If steady state error is eliminated inherently (e.g. by a PI controller), then when the control system is in a steady state, $e=0$ so that

$\theta=\theta_{r}-d_{m}$

and 
$x=\frac{\theta_{r}}{K_{p} K_{v}}-\frac{d_{m}}{K_{p} K_{v}}-\frac{K_{d} d_{p}}{K_{p} K_{v}}-\frac{d_{v}}{K_{v}}$.

If the controlled process has a Type Number of zero, then these equations can be converted to their qualitative forms:

$[\theta]=\left[\theta_{r}\right]-\left[d_{m}\right]$

and

$[x]=\frac{\left[\theta_{r}\right]}{\left\{K_{p} K_{v}\right\}}-\frac{\left[d_{m}\right]}{\left\{K_{p} K_{v}\right\}}-\frac{\left\{K_{d}\right\}\left[d_{p}\right]}{\left\{K_{p} K_{v}\right\}}-\frac{\left[d_{v}\right]}{\left\{K_{v}\right\}}$.

A qualitative variable $[z]$ is merely defined as the sign of a deviation $z$ (De Kleer \& Brown, 1984; Forbus, 1984) . Qualitative deviation $[z]$ has four values: '+' means $z$ deviates high; '-' means $z$ deviates low; ' 0 ' means $z$ has no change and '?' means the deviation of $z$ cannot be decided. $\{K\}$ represents the qualitative sign of the gain $K$, it can be viewed as an operator on a qualitative variable $[z]$ and hence doesn't exist independently. $\{K\}$ only has two values: ' + ' means $K>0$ and '-' means $K<0$.

Qualitative Equations $3 \& 4$ indicate that any change in $\theta_{r}, d_{m}, d_{p}$ and $d_{v}$ would cause the controlled variable $\theta$ and/or the controller output $x$ to deviate. By observing $[x]$ and $[\theta]$, the cause of the change, e.g. a fault or disturbance, can be detected and to some extent isolated. The latter might not be observed directly but could be reasoned from its effect on other process variables (i.e. on its descendants), Table 1 shows the knowledge for isolation. If there is ONLY one cause, then the sign or direction can be determined by the equations because, with the exception of $\left\{K_{d\}}\right\}$, the qualitative gains in the equations would be known once the control system is designed. Note that if the controlled process has a Type Number $>0$, i.e. the process is capacitive, then the proportional gains $K_{p}$ and $K_{d}$ tend to infinity and according to Equation 4, a deviation in $[x]$ is not caused by a deviation in $\left[\theta_{r}\right]$ and $\left[d_{m}\right]$ but only in $\left[d_{p}\right]$ or $\left[d_{v}\right]$. Table 1 should be modified in this case.

Similarly the following qualitative equations can be derived for the standard cascade control system shown in Fig. 3:

$$
\left[\theta_{1}\right]=\left[\theta_{r}\right]-\left[d_{m 1}\right]
$$

$$
\begin{aligned}
{\left[\theta_{2}\right] } & =\frac{\left[\theta_{r}\right]}{\left\{K_{p 1}\right\}}-\frac{\left[d_{m 1}\right]}{\left\{K_{p 1}\right\}}-\frac{\left\{K_{d 1}\right\}\left[d_{p 1}\right]}{\left\{K_{p 1}\right\}} \\
{\left[x_{1}\right] } & =\frac{\left[\theta_{r}\right]}{\left\{K_{p 1}\right\}}-\frac{\left[d_{m 1}\right]}{\left\{K_{p 1}\right\}}-\frac{\left\{K_{d 1}\right\}\left[d_{p 1}\right]}{\left\{K_{p 1}\right\}}+\left[d_{m 2}\right] \\
{\left[x_{2}\right] } & =\frac{\left[\theta_{r}\right]}{\left\{K_{p 1} K_{p 2} K_{v}\right\}}-\frac{\left\{K_{d 1}\right\}\left[d_{p 1}\right]}{\left\{K_{p 1} K_{p 2} K_{v}\right\}} \\
& -\frac{\left[d_{m 1}\right]}{\left\{K_{p 1} K_{p 2} K_{v}\right\}}-\frac{\left\{K_{d 2}\right\}\left[d_{p 2}\right]}{\left\{K_{p 2} K_{v}\right\}}-\frac{\left[d_{v}\right]}{\left\{K_{v}\right\}}
\end{aligned}
$$


Table 2 shows the rule-set that can be derived from these equations. Note also that if either the outer loop controlled process or the inner loop controlled process has a Type Number $>0$, then either $K_{p 1} \& K_{d 1}$ or $K_{p 2} \& K_{d 2}$ tend to infinity, and Equations 5$8 \&$ Table 1 should be modified accordingly.

Analysed individually, little could be deduced about a cause; the power of the approach lies in making use of knowledge about control system steady state interactions. If a controlled variable, $\theta$, in one control system deviates then it can disturb another control system. This leads to sets of coupled equations, which can be analysed simultaneously. Although certain faults, like a sensor bias, might be isolated within a SEVACS where Equations 3-4 or 5-8 would be applied to produce sets of candidates, most faults would have to be isolated centrally when all these sets are collated. This leads on to fault isolation, which is described in the next sub-section.

\subsection{Fault Isolation}

The fault isolation principle stated in italics in the next paragraph can be deduced from the basic equations derived in the previous section.

Any steady state deviation in any control system must be caused either by a fault in a loop element such as in the sensor or in the actuator/valve or by a process disturbance. Although, by the application of appropriate simple heuristic rules, it is very easy to isolate faults like a dead sensor, or a large exogenous fault or disturbance, or a sticking valve, it is more difficult to isolate faults like a sensor bias, or a small exogenous fault/disturbance. In these circumstances, the controlled variable, and its effect on descendants, is arguably the key to fault isolation: with a sensor bias, the controlled variable will deviate from its nominal value and descendants of the controlled variable will be affected; with a valve bias, or with an exogenous fault/disturbance, the controlled variable will remain at its nominal value and its descendants will not be affected.

Note that this principal holds irrespective of whether the process within the control loop has a Type number of zero or not. It was seen in the Section 2.1 that, any standard controller whose process has a zero Type Number would deviate when subjected to a sensor bias, whereas this would not happen if the Type Number $>0$. Thus, for processes with Type Numbers $>0$, a sensor bias would still affect the descendants of the respective controlled variable even though it would not cause the controlled variable, itself, to deviate.

Note also that, because control systems can interact, descendants and exogenous faults and disturbances can stem from another control system. This leads to the search and test strategy shown in Fig. 4. Starting from a deviated variable in a control system and by referring to the equations and knowledge described in the last section, a search strategy first finds all possible faults or disturbances. A test strategy then isolates those faults or disturbances that would result in the observed deviations. The search is performed by backward reasoning whilst testing is carried out by forward reasoning. All the deviated control systems are audited and the root faults can be located. Involving all control systems, this search and test strategy would be easily implemented in the Supervisor. A number of improvements can be made to the procedure to reduce the amount of search and testing (Chen, 2000). It is worth noting that obviously the Supervisor could be replaced by a human operator. 


\subsection{Identifying Interactions}

This sub-section explores how interactions can be hypothesised by performing step tests at the time control loops are commissioned. Knowledge of these interactions can lead to the determination of the steady state gains in Section 2.1. With reference to the standard control systems, there might be any of the following interactions:

(1) the interaction between a single-loop control system (or cascade outer loop) and a measured variable,

(2) the interaction between two single-loop control systems or cascade outer loops,

(3) the interaction between a cascade control system and a measured variable,

(4) the interaction between a cascade control system and a single loop control system,

(5) the interaction between two cascade control systems.

Considering each in turn. (1) Apply a step to the set-point: interaction $\underline{R}_{A B}$ can be obtained by looking at deviations in the controlled variable (A), $\underline{D}_{A}$, and in the measured variable (B), $\underline{D}_{\underline{B}}$, in the steady state. (2) Apply steps to the set-points separately: the change in one controlled variable can be viewed as a process disturbance to the other, and then $\underline{R}_{A B}$ and $\underline{R}_{B A}$ can be determined by equations derived in Section 2.1. (3) Apply a step to the outer-loop: if there is a change in the measured variable, this indicates that it is affected by this cascade control system. It is very difficult to decide whether the outer, inner, or both loops, affect this variable, so if possible, other related knowledge should then be used. For instance if the outer loop process is capacitive, it is certain that this would interact. If the outer loop process is not capacitive, for robust fault isolation, it is quite acceptable to assume it is the inner loop controlled variable that interacts, because this assumption can accommodate the other. (4) Is the same as for (3), but now the interaction is with the controlled variable as opposed to the measured variable. (5) Is similar to (4). Apply a step to the outerloop set-point: if the outer loop of the target controller deviates, then either the interaction is with the outer-loop, or the inner-loop, or both. If both, then interaction with the inner loop can be neglected because it doesn't affect the final diagnostic result. If only the inner loop controller deviates, then interaction is only with the inner loop. 


\section{Other Components of The Hybrid Strategy}

It is generally accepted that no single method is capable of isolating all possible faults and major disturbances. Hence the need for a strategy that contains a number of components. This section acknowledges the need for other components by briefly describing two, heuristic rules and governing equations. Other components would be needed, for instance, to identify a gradual drift. SEVA sensors/actuators or control loop monitoring might also be available.

\subsection{Basic Heuristic Rules}

Being based on observed changes in steady state, the approach described in Section 2 is not suited to diagnosing faults like a dead sensor, or a large exogenous fault (or disturbance), or a sticking valve, which cause the plant to 'run-away'. Such faults can be isolated by the application of appropriate simple heuristic rules. This technique has been widely adopted by many expert system based process monitoring and fault diagnosis systems and has proven to be efficient (Becraft, Guo, Lee \& Newell, 1991; Becraft \& Lee, 1993; Isermann \& Balle, 1997, 1997b; Kramer, 1987; Leonhardt \& Ayoubi, 1997).

The Benchmark is particularly amenable to a heuristic approach because of the relatively high density of instrumentation installed (Howell, Chen \& Zhang, 1997). Typical rules are like:

FOR any flow-sensor $L S$

IF the sensor-reading of $L S$ during the last 5 minutes has no value

THEN conclude that the status of LS is FAILED

Other rules can relate to the performance of individual control loops: a change in the controller's output should result in a change in the sensor-reading and also in the measurement of valve opening (if available). It is relatively easy to detect faults such as frozen/failed sensors and locked/stuck valves, by means of the simple heuristic rule:

1. examine the maximum and the minimum output of the controller during the last few minutes; if the difference between them is large enough, then perform next step;

2. examine the maximum and minimum measurement of the sensor during the last few minutes; if the difference between them is too small, then conclude that there might be something wrong with either the sensor or the valve: if the maximum is close to zero, it is most likely that the has sensor failed, otherwise either the sensor froze or the valve locked.

Some faults or disturbances can cause global changes in a plant. It is a good idea to use this piece of information to limit the number of possible candidate solutions.

\subsection{Governing Equations}

Various ambiguities that arise when applying the method in Section 2 can be eliminated by comparing with a set of fault candidates, which pertain specifically to sensor biases and leaks, and are produced by analysing a set of mathematical constraints known as governing equations [Kramer, 1987]. The basic idea is discussed here, then illustrated in the application. 
Here, governing equations describe steady state flow balances. For instance, the sum of the flows out of a process unit should equal the sum of the flows into the unit, if it is in steady state and there is no leak. If every flow rate measurement can be represented by

true flow rate + sensor bias $(B)=$ sensor reading $(\mathrm{R})$ then a governing equation can be constructed as

$\sum_{i=1}^{m}\left(R_{I i}-B_{I i}\right)=$ Leak $+\sum_{j=1}^{n}\left(R_{O j}-B_{O j}\right)$

or

$E=\sum_{i=1}^{m} R_{I i}-\sum_{j=1}^{n} R_{O j}=$ Leak $+\sum_{i=1}^{m} B_{I i}-\sum_{j=1}^{n} B_{O j}$,

where $R_{I i}$ is the sensor reading of the $\mathrm{i}^{\text {th }}$ flow in, $B_{I i}$ is the sensor bias of the $\mathrm{i}^{\text {th }}$ flow in, $R_{O j}$ is the sensor reading of the $\mathrm{j}^{\text {th }}$ flow out, $B_{O j}$ is the sensor bias of the $\mathrm{j}^{\text {th }}$ flow out, Leak is any 'loss' from the system: Leak $\geq 0$, and $E$ is the difference between the sum of sensor readings of the inputs and outputs. That is $E \approx 0$ unless there is a significant bias or leak. For instance, if $F_{I}-F_{O}=0$ represents the mass balance of a process unit, then $R_{I}-R_{O}=0$ unless there is something wrong. If the left-hand side of this expression is significantly less than zero, then the inference $\left(F_{I}-S E N S O R-B I A S-L O W\right)$ or $\left(F_{O}\right.$ SENSOR-BIAS-HIGH) can be established; if the expression is significantly greater than zero, then the inference $\left(F_{I}-S E N S O R-B I A S-H I G H\right)$ or $\left(F_{O}-S E N S O R-B I A S-\right.$ $L O W)$ or (SYSTEM-LEAK) can be established. Importantly, these conditions are the only explanations for violation of the constraint (assuming no leaks into the system). A fault candidate set can now be constructed by looking at the logical combination of these inferences. 


\section{Application to The Tennessee Eastman Process Benchmark}

The Tennessee Eastman process benchmark (Downs \& Vogel, 1993) is a simulation of a real plant that has been disguised for proprietary reasons, the flow diagram and its basic control scheme are shown in Fig. 5. The process involves the production of two products, $\mathrm{G}$ and $\mathrm{H}$, and one by-product $\mathrm{F}$, from four reactants: $\mathrm{A}, \mathrm{C}, \mathrm{D}$ and $\mathrm{E}$ :

$$
\begin{aligned}
A(g)+C(g)+D(g) & \rightarrow G(\text { liq }) & & \text { Product 1 } \\
A(g)+C(g)+E(g) & \rightarrow H(\text { Iiq }) & & \text { Product 2 } \\
A(g)+E(g) & \rightarrow F(\text { liq }) & & \text { By-product } \\
3 D(g) & \rightarrow 2 F(\text { Iiq }) & & \text { By-product }
\end{aligned}
$$

The process has five major units: a reactor, a product condenser, a vapour/liquid separator, a recycle compressor and a product stripper. The reactor is a two-phase CSTR in which exothermic, irreversible reactions occur. A non-volatile catalyst is dissolved in the liquid phase. The products have moderate volatility, and flow out of the reactor with the unreacted gases. The partial condenser recovers them from the recycle gas. The stripper minimises losses of $\mathrm{D}$ and $\mathrm{E}$ from the liquid product. Overhead from the stripper combines with the off-gas from the partial condenser for recycle to the reactor. An inert component B makes up about $0.5 \%$ of feed stream 4 . It is non-condensable and must exit in the purge (stream 9 in Fig. 5). The purge can also be used to prevent build-up of excess reactants (if any), and the byproduct $\mathrm{F}$. The plant must operate over a wide range of product composition (from a G:H ratio of 9:1, to 1:9); the amount of component $\mathrm{E}$ in the product is regulated by varying the stripper top temperature. The process has 41 measurements and 12 manipulated variables. A plant-wide decentralised control scheme has been developed by McAvoy et al. that is based on multiple single-input-single-output (SISO) control loops. Many of them are standard cascade control systems. Further details of the process and its control systems can be found in (Howell, Chen \& Zhang, 1997; McAvoy \& Ye, 1994; McAvoy, Ye \&Chen, 1995).

The Benchmark can be viewed as having 9 distributed control systems each of which can have a SEVACS associated with it (Table 3, Figs. 6 \& 7). It can be seen that SV4 is a single loop control system, SV1, SV5 and SV6 are standard cascade control systems, SV3 and SV9 are cascade control systems with two inner loops, and SV2, SV7 and SV8 look more complicated because they incorporate a common sensor. Such peculiar loops obviously weaken the argument for standardisation. However, and as will be demonstrated here, it is possible to produce a SEVACS module for each of them. In addition, there must be a finite and relatively small number of possible schemes making re-usability a practicable proposition in the longer term.

In all 28 faults or disturbances have been hypothesised for the benchmark: IDV 1 -20) from the original benchmark plus $F(1-8)$ added afterwards. IDV (8-12) and IDV(1620) are intended to assess control system performance and hence, are of no interest here. Incidents $I D V(1-7)$ involve a step change, and $F(1-8)$ involve a change in bias. Table 4 shows those considered.

\subsection{Specifying An Outline Distributed System}

Type Numbers and interactions were hypothesised by performing step tests on the process control systems' set-points one by one. It was found that the three level processes are capacitive (Type Numbers $>0$ ) because their related controller outputs don't change or deviate in steady state when their set-points' are step changed. All 
the other SEVACS related processes are open-loop stable or have an even number of unstable poles ${ }^{2}$. SEVACS interactions were hypothesised in two parts: first those SEVACS that interact were sought, then the type of interaction was established. If a set-point change in a SEVACS results in any steady state change of an element in another SEVACS, then the latter SEVACS is affected by the former. If each SEVACS is viewed as a super-node, in which all control system elements are lumped together, a branch or a link from the former super-node to the latter one can be assigned. A simple SDG representing process interactions can then be constructed by repeating this operation for all SEVACS. Fig. 8 shows that derived for the T-E benchmark. For example, the positive change of $\mathrm{A} / \mathrm{C}$ composition set-point in the Afeed flow results in steady state changes in the outputs of the reactor pressure controller (-), the product flow rate controller $(-)$, the condenser cooling controller $(+)$, the purge flow rate controller(-) and the stripper temperature controller $(+)$; it indicates that the super-node SV1 affects the super-nodes SV3, SV8, SV4, SV5 and SV9.

As discussed previously, process interactions have causal signs and can affect control systems in different ways. In addition it is sometimes sensible to describe interactions in a different way so that the right faults can be accommodated. This is particularly important here because of the prevalence of cascade control systems. For cascade control systems, and as discussed in Section 2.3, interactions can be treated as always stemming from the inner loop. Thus for example, there is a cascade arrangement in $\mathrm{SV} 1$, so the effects can be treated as stemming from the inner loop set-point $F_{A}$ (Fig. 9). Having hypothesised the causal element of SV1, those elements directly affected are now hypothesised. For instance, the outermost/main controller node $\left(\mathrm{PC}_{\mathrm{R}}\right)$ in $\mathrm{SV} 3$ has deviated, so $F_{A}$ can be viewed as an exogenous disturbance to the controlled node, reactor pressure $P_{R}$. It is also possible that $F_{A}$ is an exogenous disturbance to the two inner loops in SV3, but these effects can be ignored because if $F_{A}$ is the true fault, it can still be inferred from the change of the outermost controller output (Section 2.3). Directions can now be inferred. For instance, $F_{A}$ has a negative impact on $P_{R}$ because the positive change of $F_{A}$ causes a negative change of output of the reactor pressure controller and because of the negative proportional gain of this controller. For the same reason, $F_{A}$ has a positive impact on the product flow rate $F_{O}$ in SV8, a positive impact on the condenser cooling temperature $T_{C C W}$ in SV4, a positive impact on the purge flow rate $F_{P R G}$ in SV5 and a negative impact on the stripper temperature $T_{S T R}$ in SV9. Other effects or interactions between internal nodes in different super-nodes can be determined in a similar way.

Additional measurements, which are not within any control systems, are useful and might therefore be considered in fault isolation. These include the temperature and pressure in the separator, the pressure in the stripper, the compressor work and the recycle flow rate. These can be viewed as 'sink' nodes on the simple SDG. For instance, the following are sink nodes of SV3: separator pressure, stripper pressure.

${ }^{2}$ Both kinds of processes are analysed the same way (Chen, 2000). 


\subsection{Specification and Performance of Individual SEVACS Nodes}

This section describes the form of the SEVACS individual nodes. Standard nodes like SV1, SV4, SV5 and SV6 have already described in Section 2. This leaves the relatively standard triple loop (like SV3 \& SV9) and the special cases (SV2, SV7 \& SV8). Although not detailed, fault or disturbance directions can be determined also from the relevant equations.

Cascade Control Systems with Triple Loops: SV3, SV9

A SDG representation of SV3 (the reactor pressure, temperature cascade control system) is shown in Fig. 10. In this system, the reactor pressure $P_{R}$ is controlled by the controller $\mathrm{PC}_{\mathrm{R}}$ by manipulating the reactor temperature $T_{R}$, which is controlled by the controller $\mathrm{TC}_{\mathrm{R}}$ by manipulating the reactor cooling water temperature $T_{R C}$, which is controlled by the controller $\mathrm{TC}_{\mathrm{RC}}$ by manipulating the cooling water valve opening $V_{R} . \theta_{P R}$ is the set-point of the reactor pressure. The circled part of the system in Fig. 10 can be viewed as a virtual valve $V_{R}{ }^{\prime}$ enabling the standard cascade SEVACS analysis approach to be applied to this system.

In some circumstances the same fault or disturbance will affect different loops in $\mathrm{SV} 3$, e.g. a change in reactor level will affect both $d_{P R}$ and $d_{T R}$. In this case, the fault or disturbance would still be determined by its effect to the outer loop from node $d_{P R}$ for two reasons (see Section 2.3). Firstly, once both the outer loop and the inner loop deviate, the fault should not be from or related to the inner loop directly. Secondly, although the fault or disturbance can cause both the inner and outer loop controller to deviate, the direction of the fault should be determined by the deviation in the outer loop controller. This is because the direction of the deviation in the inner loop controller might be ambiguous so that the fault direction is not easily determined by it (Equations 7-8). A decision table is given in Table 5.

A similar approach can be taken for node SV9 (Product composition E, stripper temperature cascade control system). The $\mathrm{E}$ composition in the product, $E_{P}$, is controlled by the controller $\mathrm{EC}_{\mathrm{P}}$ by manipulating the stripper temperature $T_{P}$, which is controlled by the controller $\mathrm{TC}_{\mathrm{P}}$ by manipulating the stripper heating steam flow rate $F_{P S}$, which is controlled by the controller $\mathrm{FC}_{\mathrm{PS}}$ by manipulating the steam valve opening $V_{P S} . \theta_{E P}$ is the set-point of the E composition in the product. A decision table is given in Table 6.

Non-Standard Control Systems: SV2

$\mathrm{SV} 2$ focuses on the $\mathrm{G} / \mathrm{H}$ composition, reactor level control system. Fig. 11 shows a block diagram representation that is pseudo-linear. Four controllers, two valves and four processes are involved. Controller output $x_{0}$ represents the demanded ratio of the flow rate of $\mathrm{D}\left(y_{1}\right)$ to the flow rate of $\mathrm{E}\left(y_{2}\right)$. This ratio is important for two reasons: to maintain the $\mathrm{G} / \mathrm{H}$ composition ratio in the final product $\theta_{0}$ at its set-point $\theta_{r 0}$, and to maintain the reactor level $\theta$ at the set-point $\theta_{r}$. Variables $d_{m 0}, d_{m l}, d_{m 2}$ and $d_{m}$ represent respective sensor biases, $d_{v 1}$ and $d_{v 2}$ represent respective valve biases, $d^{\prime}{ }_{p 0}$, $d_{p 1}^{\prime}, d_{p 2}^{\prime}$ and $d_{p}$ represent respective process disturbances. Parameter $K_{d}$ is the steady state gain of the level process disturbance transfer function $G_{d}(S)$, similar to $K_{p}$, it also tends to infinity. All variables represent deviations from their nominal values.

The circled part in Fig. 11 can be viewed as the D/E flow ratio (r) control system, which can then be viewed as the inner loop of the cascade system shown in Fig. 12. 
Linearising the equations about $\mathrm{D} / \mathrm{E}$ flow operating point leads to $r=\alpha y_{1}-\beta y_{2}$ and $d_{m r}=\alpha d_{m 1}-\beta d_{m 2}(\alpha>0, \beta>0)$. Because $G_{p}(s)$ is a capacitive process, at a steady state, it then follows that

$$
\begin{aligned}
& x_{0}=\alpha d_{m 1}-\beta d_{m 2}+r \\
& x=y_{2}+d_{m 2} \\
& x_{1}=\frac{y_{1}-d_{p 1}^{\prime}-K_{p 1} \cdot d_{v 1}}{K_{p 1} \cdot K_{v 1}} \\
& x_{2}=\frac{y_{2}-d_{p 2}^{\prime}-K_{p 2} \cdot d_{v 2}}{K_{p 2} \cdot K_{v 2}}
\end{aligned}
$$

Some observations that can be derived from these equations are summarised in Table 7. It can be seen that the change of $d_{v 1}$ and $d^{\prime}{ }_{p 1}$ can only cause $x_{1}$ to change and the change of $d_{v 2}$ and $d_{p 2}^{\prime}$ can only cause $x_{2}$ to deviate, because all these variables are in the innermost loops in the control system.

\section{Non-Standard Control Systems: SV7/SV8}

Nodes SV7 and SV8 have to be considered together because product flow rate appears in both. SV7 represents the stripper level, product flow rate cascade control system. SV8 represents the product flow rate, C flow rate cascade control system. Fig. 13 shows a block diagram for these two control systems and roughly in which the upper part represents SV8 and the lower one represents SV7. $\theta_{1,1}$ and $\theta_{1,2}$ represent the product flow rate and the stripper level, $\theta_{r, 1}$ and $\theta_{r, 2}$ are their set-points respectively. Although SV7 and SV8 are two standard cascade control systems, they have a common controlled variable, product flow rate $\theta_{1,1}$, which is the inner loop controlled variable in SV7 as well as the outer loop controlled variable in SV8. As such these two interact and should not be considered separately.

In SV7, the stripper level is actually controlled by manipulating the product flow rate out of the stripper through processes $G_{z 11}(s)$ and $G_{z 22}(s)$ with steady state gains $K_{z 11}$ and $K_{z 22}$ respectively, and the $\mathrm{C}$ flow rate into the stripper through the process $G_{z 12}(\mathrm{~s})$ with a steady state gain $K_{z 12}$. In SV8, the product flow rate is actually controlled by manipulating the level of the stripper through processes $G_{z 12}(s)$ and $G_{z 21}(s)$ with steady state gains $K_{z 12}$ and $K_{z 21}$ respectively, and the product valve opening $\mathrm{y}_{2,2}$ through $G_{z 11}(s)$ with a steady state gain $K_{z 11}$.

In SV8, $x_{1,1}$ and $x_{2,1}$ are the outputs of the outer loop controller $G_{c 11}(s)$, with the proportional gain $K_{c l, 1}$, and the inner loop controller $G_{c 21}(s)$, with the proportional gain $K_{c 2,1} . \quad e_{1,1}$ and $e_{2,1}$ represent the control errors to these two controllers respectively. $d_{v, 1}, d_{p 2,1}^{\prime}, d_{p 1,1}^{\prime}, d_{m 2,1}$ and $d_{m 1,1}$ represent the bias of the valve $G_{v 1}(s)$ with the steady state gain $K_{v, 1}$, the disturbance to the inner loop process $G_{p 21}(s)$, with the steady state gain $K_{p 2,1}$, the disturbance to the product flow rate related processes $G_{z 11}(s)$ and $G_{z 21}(s)$, with steady state gains $K_{z 1,1}$ and $K_{z 2,1}$ respectively, the inner loop sensor bias and the outer loop sensor bias in SV8, in which $d_{m 1,1}$ is also as the inner loop sensor bias in SV7.

In SV7, $x_{1,2}$ and $x_{2,2}$ are the outputs of the outer loop controller $G_{c 12}(s)$, with the proportional gain $K_{c l, 2}$, and the inner loop controller $G_{c 22}(s)$, with the proportional 
gain $K_{c 2,2} . e_{1,2}$ and $e_{2,2}$ represent the control errors to these two controllers respectively. $d_{v, 2}, d_{p 1,2}$ and $d_{m 1,2}$ represent the bias of the valve $G_{v 2}(s)$ with the steady state gain $K_{v, 2}$, the disturbance to the level related processes $G_{z 12}(s)$ and $G_{z 22}(s)$, with steady state gains $K_{z 1,2}$ and $K_{z 2,2}$ respectively, and the level sensor bias in SV7. $G_{d 12}(s)$ with a steady state gain $K_{d 1,2}$ represents a disturbance process from $d_{p 1,2}$ to the level $\theta_{1,2}$. Note that the level related processes are capacitive, which means $G_{z 12}(s), G_{z 22}(s)$ and $G_{d 12}(s)$ have the same denominator and their respective steady state gains $K_{z 1,2} \rightarrow$ $+\infty, \mathrm{K}_{\mathrm{z} 2,2} \rightarrow-\infty$ and $K_{d 1,2} \rightarrow \pm \infty$. However, any ratio between two of these three steady state gain should be finite. Thus following qualitative equations can be derived:

$$
\begin{aligned}
{\left[x_{1,1}\right] } & =\left[\theta_{r, 1}\right]-\left[d_{m 1,1}\right]-\left\{\frac{K_{d 1,2}}{K_{z 1,2}}\right\} d_{p 1,2}+\left[d_{m 2,1}\right] \\
{\left[x_{2,1}\right] } & =\frac{\left[\theta_{r, 1}\right]}{\left\{K_{p 2,1} K_{v, 1}\right\}}-\frac{\left[d_{m 1,1}\right]}{\left\{K_{p 2,1} K_{v, 1}\right\}}-\frac{\left[d_{p 2,1}^{\prime}\right]}{\left\{K_{p 2,1} K_{v, 1}\right\}}-\left\{\frac{K_{d 1,2}}{K_{p 2,1} K_{v, 1} K_{z 1,2}}\right\}\left[d_{p 1,2}\right]-\frac{\left[d_{v 1}\right]}{\left\{K_{v, 1}\right\}} \\
{\left[x_{1,2}\right]=} & {\left[\theta_{r, 1}\right] } \\
{\left[x_{2,2}\right]=} & \frac{\left[\theta_{r, 1}\right]}{\left\{K_{v, 2} K_{z 1,1}\right\}}-\left\{\frac{K_{z 2,1}}{K_{v, 2} K_{z 1,1}}\right\}\left[\theta_{r, 2}\right]-\frac{\left[d_{m 1,1}\right]}{\left\{K_{v, 2} K_{z 1,1}\right\}} \\
& +\left\{\frac{K_{z 2,1}}{K_{v, 2} K_{z 1,1}}\right\}\left[d_{m 1,2}\right]-\frac{\left[d_{p 1,1}^{\prime}\right]}{\left\{K_{v, 2} K_{z 1,1}\right\}}-\frac{\left[d_{v 2}\right]}{\left\{K_{v, 2}\right\}}
\end{aligned}
$$

Based on the above qualitative equations, a decision table for fault detection and diagnosis can be made (Table 8). Directions of various faults or disturbances can be also determined from these equations. 


\section{Some Results}

The effects of the various faults and disturbances were observed by simulating each in turn and looking at the long-term (i.e. pseudo steady state) responses. Sections 5.1 and 5.2 describe how two of these incidents, $I D V(1)$ and $F(3)$, would be analysed by the steady state based procedures. Section 5.3 then looks more generally at the performance of the SEVACS when subjected to any of the faults and disturbances. Section 5.4 describes, briefly, how governing equations might be incorporated into the Supervisor.

\subsection{IDV(1): Lower A/C Feed Ratio Whilst Keeping the Composition of B in Stream 4 Constant}

In the steady state, SV1, SV5 and SV8 will be affected. SV1: the A, C composition ratio $(\mathrm{A} / \mathrm{C})$ controller and the $\mathrm{A}$ feed flow rate controller have positive deviations. SV5: the purge B composition controller and the purge rate controller have negative deviations. SV8: the product flow rate controller and the $\mathrm{C}$ feed flow rate controller have negative deviations. From Fig. 8 and by applying the fault isolation principle, the following relevant sub-set can be obtained: $\left\{d_{m l}(\mathrm{SV} 8)\right\} \subset \mathrm{D}_{\mathrm{p} 1}(\mathrm{SV} 1)$. Here $d_{m l}(\mathrm{SV} 8)$ is used to denote the deviation in the sensor bias in the outer loop of SV8 and $\mathrm{D}_{\mathrm{p} 1}(\mathrm{SV} 1)$ is used to denote the set of disturbances that pertain to the outer loop of SV1.

If a fault isolation procedure is applied then:

search process: starting from the super-node SV1, $d_{m l}(\mathrm{SV} 1)$ low and $\mathrm{D}_{\mathrm{p} 1}(\mathrm{SV} 1)$ low would form a fault candidate set because of the outer-loop controller deviation; since $\left\{d_{m l}(\mathrm{SV} 8)\right\} \subset \mathrm{D}_{\mathrm{p} 1}(\mathrm{SV} 1)$ and because of the sign of the interaction (Fig. 9), $d_{m l}$ (SV8) high would then be appended;

test process: from Fig. $8, d_{m l}(\mathrm{SV} 1)$ low would be rejected because its descendants in SV1, SV3, SV4, SV5, SV8 and SV9 should deviate; $d_{m l}(\mathrm{SV} 8)$ high would be rejected because, on the basis of the fault isolation principal, it should cause SV1, SV2, SV3, SV4, SV5, SV6, SV8 and SV9 to deviate. As it stands set $\mathrm{D}_{\mathrm{p} 1}(\mathrm{SV} 1)$ is nominally empty because it doesn't contain 'unknown' disturbances. Further knowledge is required. For instance if a more detailed SDG representation of the process is available then this could be used to generate more hypotheses. Alternatively a list of likely causes (e.g. as in Table 5) might be available, which could be used in a simulation to predict the various effects. Another possibility is that, having been notified that a fault or disturbance to nodes SV1, SV5 \& SV8 has arisen with candidate set $\mathrm{D}_{\mathrm{p} 1}(\mathrm{SV} 1)$ low, the operator might provide his own hypotheses. Suppose that he was to hypothesise IDV(1) \& IDV(2), and these were simulated to obtain steady state effects. Then IDV(2) would be rejected because it would be seen to cause SV1, SV2, SV3, SV5, SV8 and SV9 to deviate. This would leave IDV(1), which can cause SV1, SV5 and SV8 to deviate in the above directions.

Some of the deviations sought by the search process didn't actually happen in the simulation. However sufficient super-nodes did deviate to enable the process to eliminate the alternatives. Also it is worth pointing out that IDV(1) is just one disturbance, other disturbances might exist that have similar effects and this possibility should not be ruled out. It is therefore important that a dialogue takes place with the operators to ensure that the system 'learns'. 


\subsection{F(3): Reactor-Level-Sensor-Bias-Low}

In the steady state, although the fault actually occurred in SV2, this would not be apparent, locally, because the level process is capacitive and nothing wrong would be observed in SV2. However SV3, SV4, SV5, and SV9 will be affected. SV3: the reactor pressure controller and the reactor temperature controller have positive deviations, and the reactor cooling controller has a negative deviation. SV4: the condenser cooling controller has a negative deviation. SV5: the purge flow rate controller has a positive deviation. SV9: the stripper temperature controller and the stripper steam controller have negative deviations. The additional measurements, the compressor work and the recycle flow rate will also deviate, negatively.

Search process: starting from the super-node SV3, the fault candidate set would be $\left\{d_{m l}(\mathrm{SV} 3)\right\} \cup \mathrm{D}_{\mathrm{p} 1}(\mathrm{SV} 3) \supset\left\{d_{m l}(\mathrm{SV} 1), d_{m l}(\mathrm{SV} 2), d_{m l}(\mathrm{SV} 4), d_{m l}(\mathrm{SV} 5), d_{m l}(\mathrm{SV} 8)\right\}$. Note that this contains the outer loop sensor bias for SV2 i.e. reactor-level-sensorbias because the reactor level process is capacitive and hence the sensor bias can be viewed as a process disturbance to SV3.

Test process: $d_{m l}(\mathrm{SV} 3)$ can be rejected because additional measurements, the separator pressure and the stripper pressure, don't deviate. Bias $d_{m l}$ (SV4) can be rejected because it should lead to deviations in SV2, SV6 and SV8; biases $d_{m l}(\mathrm{SV} 1)$ and $d_{m l}(\mathrm{SV} 8)$ can be rejected because neither SV1 nor SV8 deviate; bias in $d_{m l}(\mathrm{SV} 5)$ can be rejected because it should lead to a deviation in SV8. This leaves the bias $d_{m l}$ (SV2) i.e. the reactor-level-sensor-bias, which can cause SV3, SV4, SV5 and SV9 to deviate in the observed directions.

Once again it is unlikely that the observed effects can be attributed, uniquely, to one fault, other disturbances or faults might affect $\mathrm{D}_{\mathrm{p} 1}(\mathrm{SV} 3)$. It is therefore important that a dialogue takes place with the operators and that any new scenarios are recorded to improve the performance of the system. Suppose that $I D V(2)$ has been previously stored in the knowledge-base: since there are no deviations in SV2 and $I D V(2)$ is a disturbance to SV2, IDV(2) would be rejected.

\subsection{SEVACS Performance in General}

Table 9 was constructed by simulating the various faults and disturbances and observing the steady state changes in the controller outputs. An ' $\mathrm{H}$ ' is used to indicate those incidents that could not be diagnosed by applying a steady state approach but could be diagnosed by applying heuristic rules instead. Slow drift, IDV(13), would not be diagnosed. The results can also be represented by sub-sets associated with the various process disturbances and sensor biases and this information could be stored in a knowledge-base. For instance, for node SV1: $\{I D V(1), I D V(2), F(5)\} \subset \mathrm{D}_{\mathrm{p} 1}(\mathrm{SV} 1), I D V(6) \subset \mathrm{D}_{\mathrm{p} 2}(\mathrm{SV} 1), d_{m 1}(\mathrm{SV} 1)=\mathrm{A} / \mathrm{C}$ composition analyser bias, $d_{m 2}(\mathrm{SV} 1)=\mathrm{A}$ flow rate sensor bias and for node SV5: $\{I D V(1), I D V(2)$, $F(5)\} \subset \mathrm{D}_{\mathrm{p} 1}(\mathrm{SV} 5),\{F(2), F(3), F(4)\} \subset \mathrm{D}_{\mathrm{p} 2}(\mathrm{SV} 5), d_{m 1}(\mathrm{SV} 5)=F(1)$ and $d_{m 2}(\mathrm{SV} 5)=$ purge flow rate sensor bias.

Although Table 9 shows that there is merit in combining the outputs from the SEVACS, it doesn't say much else. Most of the important information is contained in the various sub-sets. For instance, superficially, $I D V(3), I D V(4), \& F(4)$ appear to affect SV3 the same way but in practice they affect the control system at different nodes: $I D V(4)$ is a disturbance to the innermost loop $\left(d_{T R C}\right), I D V(3)$ is a disturbance to 
the second inner loop $\left(d_{T R}\right)$, whereas $F(3)$ causes a disturbance to the outermost loop $\left(d_{P R}\right)$. Another interesting point is with $I D V(2): I D V(2) \in \mathrm{D}_{\mathrm{p}}(\mathrm{SV} 2)$ and $I D V(2) \in$ $\mathrm{D}_{\mathrm{p} 0}^{\prime}(\mathrm{SV} 2)$ i.e. it causes a different 'pattern' of controller outputs to deviate.

\subsection{Governing Equations}

Faults $F(5)-F(7)$ would violate certain governing equations and hence could be detected using a combination of governing equations with SEVACS. The approach is first to generate a set of candidates by analysing the appropriate governing equations (Howell, Chen \& Zhang, 1997); various possibilities are then eliminated by referring to SEVACS knowledge. If the material accumulations in the vessels are ignored, then the following mass balance equations can be obtained provided that there are additional measurements for Stream 5 and Stream 7:

$$
\begin{aligned}
& \text { Stream } 1+\text { Stream } 2+\text { Stream } 3+\text { Stream } 5+\text { Stream } 8-\text { Stream6 }=0 \\
& \text { Stream6 }- \text { Stream } 8-\text { Stream } 9-\text { Stream } 10=0 \\
& \text { Stream } 4+\text { Stream } 10-\text { Stream } 5-\text { Stream } 11=0 \\
& \text { Stream } 1+\text { Stream } 2+\text { Stream } 3+\text { Stream } 4-\text { Stream } 9-\text { Stream } 11=0 \\
& \text { Stream6 }- \text { Stream } 7=0
\end{aligned}
$$

Values, averaged over the relatively short period time, say 5 minutes, were used to overcome random deviations, disturbances and uncertainties in the process. In addition, the benchmark's 'chemical' units of flow rate $\left(\mathrm{kscmh}\right.$ and $\left.\mathrm{m}^{3} / \mathrm{h}\right)$ were changed to $\mathrm{kg} / \mathrm{h}$ to perform mass balances. Any of the constraints were then deemed to be in conflict if their error exceeded $5 \%$ of the total mass either into or out of the node. Every 5 minutes a 16-element vector was generated for each equation, which described that which could be deduced from that equation at that time. The first 11 elements pertained to the states of the streams, whilst the last five represented a leak from each unit; $\mathrm{N}$ denoted normality, $\mathrm{H}$ denoted high, $\mathrm{L}$ denoted low and $\mathrm{U}$ denoted undetermined; the subscript denoted likeliness of the fault, the bigger the number, the more likely the fault. For instance Equation 20 might have suggested (Stream6-BIAS$L O W$ ) or (Stream8-BIAS-HIGH) or (Stream9-BIAS-HIGH) or (Stream10-BIAS$H I G H$ ), which would be output as $\left[\mathrm{U}_{1} \mathrm{U}_{1} \mathrm{U}_{1} \mathrm{U}_{1} \mathrm{U}_{1} \mathrm{~L}_{1} \mathrm{U}_{1} \mathrm{H}_{1} \mathrm{H}_{1} \mathrm{H}_{1} \mathrm{U}_{1} \mathrm{~N}_{1} \mathrm{~N}_{1} \mathrm{~N}_{1} \mathrm{~N}_{1}\right.$ U1]. These statements were then combined by applying the operator defined in Table 10 , where $\mathrm{R}$ is the ambiguous result $\mathrm{L}^{\wedge} \mathrm{H}$. +For example, suppose that the governing equations pertaining to Equations 20 and 21 are violated resulting in the output of governing vectors $\mathrm{v} 1, \mathrm{v} 2, \ldots$, and $\mathrm{v} 5$ :

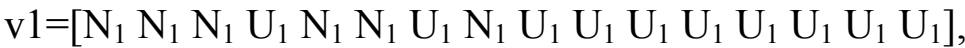

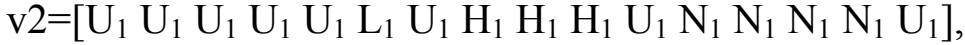

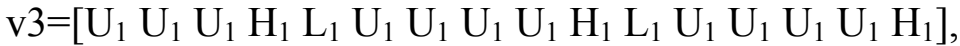

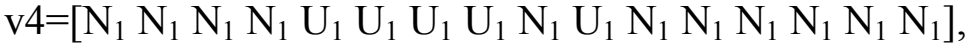

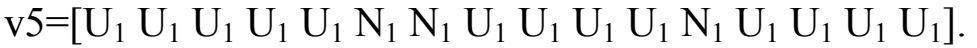

Then,

$$
\mathrm{v} 1^{\wedge} \mathrm{v} 2^{\wedge} \mathrm{v} 3^{\wedge} \mathrm{v} 4^{\wedge} \mathrm{v} 5=\left[\mathrm{N}_{1} \mathrm{~N}_{1} \mathrm{~N}_{1} \mathrm{~N}_{1} \mathrm{~N}_{1} \mathrm{~N}_{1} \mathrm{~N}_{1} \mathrm{~N}_{1} \mathrm{~N}_{1} \mathrm{H}_{2} \mathrm{~N}_{1} \mathrm{~N}_{1} \mathrm{~N}_{1} \mathrm{~N}_{1} \mathrm{~N}_{1} \mathrm{~N}_{1}\right]
$$

The $10^{\text {th }}$ element of the vector (Equation 24) indicates that the sensor value of stream 10 is most likely to have failed high.

If fault $F(5)$ happened, then the governing Equations 21 and 22 would be violated whilst the others would not. Thus according to the logical operation, C-flow-ratesensor-bias-high (stream 4), product-flow-rate-sensor-bias-low (stream 11) and the system leak would form a fault candidate set. Because $\mathrm{C}$ flow rate sensor is in the 
inner loop of a cascade system and referring to SEVACS knowledge in Section 2, this sensor bias shouldn't cause the inner loop controller to deviate and thus this sensor bias would be rejected.

If fault $F(6)$ happened, the governing Equations 19 and 22 would be violated whilst the others would not. Then A-flow-rate-sensor-bias-high (stream 1), D-flow-ratesensor-bias-high (stream 2), E-flow-rate-sensor-bias-high (stream 3) and the system leak would form a fault candidate set. These three flow rate sensors are all in inner loops, and according to Section 2, any inner loop sensor bias would cause the respective outer loop controller to deviate, thus both A-flow-rate-sensor-bias-high (stream 1) and E-flow-rate-sensor-bias-high (stream 3) would be rejected.

Fault $F(7)$ is very similar to the fault $F(6)$ and would be diagnosed similarly. 


\section{Conclusions}

Although the broad objective of this paper has been to show how a distributed hybrid strategy might be applied to the isolation of faults and disturbances in the Tennessee Eastman Process, the main emphasis has been on the application of a steady state based approach. It is intended that the strategy would build on existing structures for distributed control systems, so would be easy to implement, cheap and widely applicable. Configuring would be performed in parallel with control loop tuning: step tests would probably be needed to identify interactions between control systems. The steady state approach is qualitative model based and can work, to some extent with a minimal amount of knowledge. A way in which a knowledge-base might evolve to improve isolation capabilities has been suggested in Section 5. Knowledge is the key to isolation and the role of the operator has been briefly discussed.

Being steady state based, there is clearly an issue of actually knowing when such a state is obtained. In fact with the application here, and in order to minimise delays in reaching a decision, the process was often assumed to be in a steady state when it was clearly on some long-term, asymptotic trajectory. Although Chen \& Howell (2001) have identified possible algorithms, further investigation is still needed to make the approach practicable. Even when all the control loops attained a 'steady state', it was sometimes difficult to detect small changes. Methods dealing with the possibility of a certain deviation not being observed need to be considered also.

To meet the objective of ease of implementation, reliance must be placed on standard templates. It has been described how the T-E process would require a number of 'non-standard' templates because it has a few unusual control systems. However these have been produced relatively easily and if adopted by a commercial vendor, it is clear that a reasonably extensive library could soon be developed. Another issue worthy of consideration is that of a plant refurbishment. Following a plant refurbishment it would be quite normal for the controllers to be re-tuned. Revised information would be obtained as part of this activity, which would be used to reconfigure the SEVACS modules. 


\section{References}

Alonso, G.C., Pulido, J.B., and Acosta, G. (1998). Online industrial diagnosis: An attempt to apply artificial intelligence techniques to process control, In: Mira, J., A. P. del Pobil, M. Ali (Eds.) Methodology and Tools in Knowledge-Based Systems, Lecture Notes in Artificial Intelligence, Springer, pp. 804-813.

Ayoubi, M. and Isermann, R. (1996). Fuzzy system design based on a hybrid neural structure and application to the fault diagnosis of technical processes, Control Engineering Practice, 4, pp. 35-42.

Basseville, M. and Nikiforov, I.V. (1993). Detection of Abrupt Changes - Theory and Application, Prentice Hall.

Becraft, W.R., Guo, D.Z., Lee, P.L. and Newell, R.B. (1991). Fault diagnosis strategies for chemical plants: a review of competing technologies, Proc. $4^{\text {th }}$ Int. Symp. Process Systems Engineering, Montebello, Canada, II, pp. 12.1-12.15.

Becraft, W.R. and Lee, P.L. (1993). An integrated neural network/expert system approach to fault diagnosis, Computers and Chemical Engineering, 17, pp. 10011014.

Chantler, M.J., Coghill, G. M., Shen, Q., and Leitch, R.R. (1998). Selecting tools and techniques for model-based diagnosis, Artificial Intelligence in Engineering, 12, pp 81-98.

Chen J. (2000). Control System Based Loop and Process Monitoring, PhD Thesis, University of Glasgow.

Chen, J. and Howell, J. (2001). A self-validating control system based approach to plant fault detection and diagnosis, Computers and Chemical Engineering, 25, pp 337-358.

Chow, E.Y. and Willsky, A.S. (1984). Analytical redundancy and the design of robust failure detection systems, IEEE Transactions on Automatic Control, AC-29, pp.603-614.

Clarke, D.W. (1995). Sensor, actuator, and loop validation, IEEE Control Systems Magazine, August, pp. 39-45.

Dorf, R.C. and Bishop, R.H. (1995). Modern Control Systems, 7th ed., Addison Wesley.

Downs, J.J. and Vogel, E.F. (1993). A Plant-Wide Industrial Process Control Problem, Computers and Chemical Engineering, 17, pp. 245-255.

Forbus, K.D. (1984). Qualitative process theory, Artificial Intelligence, 24, pp 85-168.

Gertler, J. (1993). Survey of model-based failure detection and isolation in complex plants, IEEE Control Systems Magazine, 8(3), pp. 3-11.

Gertler, J. (1998). Fault Detection and Diagnosis in Engineering Systems, Marcel Dekker.

Harris, T. (1989). Assessment of control loop performance, Canadian Journal of Chemical Engineering, 67, pp 856-861

Henry, M.P. and Clarke, D. W. (1993). The self-validating sensor: rationale, definitions and examples, Control Engineering Practice, 1, pp. 585-610. 
Himmelblau, D.M. (1978). Fault Detection and Diagnosis in Chemical and Petrochemical Processes, Elsevier Scientific Publishing Company.

Howell, J., Chen, J. and Zhang, J. (1997). The Tennessee Eastman Problem as a process monitoring benchmark, IFAC Safeprocess'97, Hull, UK, pp. 222-227.

Iri, M. K., Aoki, K., O’Shima, E., \& Matsuyama, H. (1979). An algorithm for diagnosis for system failure in the chemical process, Computers \& Chemical Engineering, 3, 489-493.

Isermann, R. (1984). Process fault detection based on modeling and estimation methods - A survey, Automatica, 20 (1984) 387-404.

Isermann, R. (1997). Supervision, fault-detection and fault-diagnosis methods - An introduction, Control Engineering Practice, 5, pp.639-652.

Isermann, R. and Ballé, P. (1997). Trends in the application of model-based fault detection and diagnosis of technical processes, Control Engineering Practice, 5, pp.709-719.

de Kleer, J. and Brown, J.S. (1984). Qualitative physics based on confluences, Artificial Intelligence, 24, pp 7-83.

Kramer, M.A. (1987). Malfunction diagnosis using quantitative models with nonboolean reasoning in expert systems, AIChE J., 33, pp. 130-140.

Kramer, M.A. and Palowitch, B. L. (1987). A Rule-Based Approach to Fault Diagnosis Using the Signed Directed Graph, AIChE J., 33, pp. 1067-1078.

Kutsuwa, Y., Kojima, K., and Matsuyama, H. (1988). Fault diagnosis of a batch process by use of pattern-recognition technique, Kagaku Kogaku Ronbunshu, 14, 20-25.

Leonhardt, S. and Ayoubi, M. (1997). Methods of fault diagnosis, Control Engineering Practice, 5, pp. 683-692.

Lunze, J., and Schiller, F. (1999). Example of fault diagnosis by means of probabilistic logic reasoning, Control Engineering Practice, 7, pp 271-278.

McAvoy, T.J. and Ye, N. (1994). Base Control for the Tennessee Eastman Problem, Computers and Chemical Engineering, 18, pp. 383-413.

McAvoy. T.J., Ye, N. and Chen, G. (1995). An Improved Base Control for the Tennessee Eastman Problem, Proceedings of American Control Conference, WA9, 1995, pp. 240-244.

Mo, K. J., Lee, G., Nam, D. S., Yoon, Y. H., and Yoon, E. S. (1997). Robust fault diagnosis based on clustered symptom trees, Control Engineering Practice, 5, 199-208.

Shiozaki, J., Matsuyama, H., Tano, K., and O’Shima, E. (1984). Diagnosis of chemical processes by use of signed directed graphs - extensions to 5-range patters of abnormality, Kagaku Kogaku Ronbunshu, 10, 233-239.

Thornhill, N. F., Sadowski, R., Davis, J.R., Fedenczuk, P., Knight, M.J., Prichard, P. and Rothenberg, D. (1996). Practical experiences in refinery control loop performance assessment, IEE Conference Publication, 427/1, pp 175-180. 
Thornhill, N. F., Oettinger, M. and Fedenczuk, P. (1999). Refinery-wide control loop performance assessment, Journal of Process Control, 9, pp 109-124.

Tsuge, Y., Shiozaki, J., Matsuyama, H., O’Shima, E., Iguchi, Y., Fuchigami, M., and Matsushita, M. (1984a). Feasibility study of fault diagnosis system for chemical plants, Kagaku Kogaku Ronbunshu, 10, 240-246.

Tsuge, Y., Shiozaki, J., Matsuyama, H., O’Shima, E., Iguchi, Y., Fuchigami, M., and Matsushita, M. (1984b). Improved method of display in the fault diagnosis system - utilization of information about delays among state variables, Kagaku Kogaku Ronbunshu, 10, 531-534.

Tyler, M. L. and Morari, M. (1996). Performance monitoring of control systems using likelihood methods, Automatica, 32, pp 1145-1162.

Vedam, H. and Venkatasubramanian, V. (1999). PCA-SDG based process monitoring and fault diagnosis, Control Engineering Practice, 7, 903-917.

Wang, X.Z., Yang, S.A., Veloso, E., Lu, M.L. and McGreavy, C. (1995). Qualitative process monitoring - a fuzzy signed directed graph method, Computers and Chemical Engineering, 19, Suppl, S735-S740. 